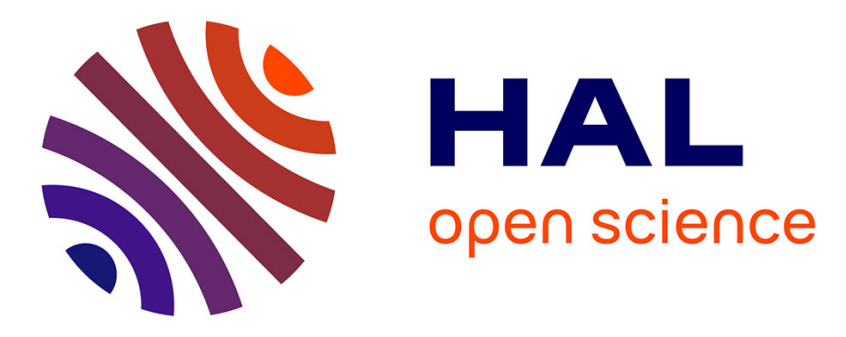

\title{
Viburnum tinus Fruits Use Lipids to Produce Metallic Blue Structural Color
}

\author{
Rox Middleton, Miranda Sinnott-Armstrong, Yu Ogawa, Gianni Jacucci, \\ Edwige Moyroud, Paula Rudall, Chrissie Prychid, Maria Conejero, Beverley \\ Glover, Michael Donoghue, et al.
}

\section{To cite this version:}

Rox Middleton, Miranda Sinnott-Armstrong, Yu Ogawa, Gianni Jacucci, Edwige Moyroud, et al.. Viburnum tinus Fruits Use Lipids to Produce Metallic Blue Structural Color. Current Biology - CB, 2020, 30 (19), pp.3804-3810.e2. 10.1016/j.cub.2020.07.005 . hal-02977848

\section{HAL Id: hal-02977848 \\ https://hal.science/hal-02977848}

Submitted on 14 Nov 2020

HAL is a multi-disciplinary open access archive for the deposit and dissemination of scientific research documents, whether they are published or not. The documents may come from teaching and research institutions in France or abroad, or from public or private research centers.
L'archive ouverte pluridisciplinaire HAL, est destinée au dépôt et à la diffusion de documents scientifiques de niveau recherche, publiés ou non, émanant des établissements d'enseignement et de recherche français ou étrangers, des laboratoires publics ou privés. 
1 Viburnum tinus Fruits Use Lipids to produce Metallic Blue Structural

2 Colour

3 Rox Middleton ${ }^{\dagger 1,8}$, Miranda Sinnott-Armstrong ${ }^{\dagger 2,9}$, Yu Ogawa $^{3}$, Gianni Jacucci ${ }^{1}$, Edwige Moyroud ${ }^{4,5}$, Paula J.

4 Rudall $^{6}$, Chrissie Prychid ${ }^{6}$, Maria Conejero ${ }^{6}$, Beverley J. Glover ${ }^{7}$, Michael J. Donoghue ${ }^{2}$, Silvia Vignolini ${ }^{*}$

$5 \quad{ }^{\dagger}$ Shared co-first authorship

$6 \quad{ }^{1}$ Chemistry Department, University of Cambridge, Lensfield Road, Cambridge CB2 1EW, UK

$7 \quad{ }^{2}$ Department of Ecology and Evolutionary Biology, Yale University, PO Box 208106, New Haven, CT 06520, USA

$8{ }^{3}$ Univ. Grenoble Alpes, CNRS, Cermav, Grenoble 38000, France

$9 \quad{ }^{4}$ The Sainsbury Laboratory, University of Cambridge, 47 Bateman Street, Cambridge CB2 1LR, UK

$10{ }^{5}$ Department of genetics, University of Cambridge, 20 Downing PI, Cambridge CB2 3EJ, UK

$11{ }^{6}$ Royal Botanic Gardens, Kew, Richmond, Surrey TW9 3AB, UK

$12{ }^{7}$ Department of Plant Sciences, University of Cambridge, Downing Street, Cambridge CB2 3EA, UK

$13{ }^{8}$ Current Address: Department of Biological Sciences, University of Bristol, 24 Tyndall Ave, Bristol, BS81TQ, UK

$14{ }^{9}$ Current Address: Department of Ecology and Evolutionary Biology, University of Colorado-Boulder, 80309 USA

15 *Lead contact: sv319@cam.ac.uk

16

17

\section{Summary}

Viburnum tinus is an evergreen shrub that is native to the Mediterranean region but cultivated widely in Europe and around the world. It produces ripe metallic blue fruits throughout winter [1]. Despite its limited fleshy pulp,[2] its high lipid content[3] makes it a valuable resource to the small birds[4] that act as its seed-dispersers[5]. Here, we find that the metallic blue appearance of the fruits is produced by globular lipid inclusions arranged in a disordered multilayer structure. This structure is embedded in the cell walls of the epicarp and underlaid with a dark layer of anthocyanin pigments. The presence of such large, organised lipid aggregates in plant cell walls represents a new mechanism for structural colouration and may serve as an honest signal of nutritional content.

\section{Results and Discussion}

The colours of fleshy fruits are thought to serve primarily to attract animal dispersers, and understanding the origins and diversity of fleshy fruit colours provides insight into the ecological interactions between plants and their dispersers. [7] Viburnum tinus (Adoxaceae) is a shrub native to the Mediterranean region that is now cultivated around the world.[8] It produces large numbers of metallic blue fruits throughout the year. Although a variety of bird dispersers consume $V$. tinus fruits, [2] these serve as an especially important food source for birds during winter, including the Eurasian blackcap (Sylvia atricapilla)[9] and the European robin (Erithacus rubecola).[10] While the remarkable blue metallic appearance of $V$. tinus fruits (Figure 1 ) is commonly known and previously reported,[11] the mechanism by which such blue colour is produced has not been elucidated. In a review of earlier literature,[12] the colour of $V$. tinus has been attributed to the presence of anthocyanin pigments.

The fruits of $V$. tinus (Figure 1c) reflect light directionally (producing its metallic appearance) in the blue and UV spectral region (Figure S1). The polarisation of the reflected light is mostly retained (Figure S2a-c\&e), a property which indicates that the colouration is structural, rather than pigmentbased, originating in reflection from the highly structured cell wall of the outer epicarp (Figure 2a). Dissection of this tissue releases a dark red anthocyanin pigment.[12] Light that is not reflected by the photonic structure is absorbed by the dark red pigment underneath (Figure 2A, Figure 3C \& Figure S3E) This absorption prevents backscattering of light, increasing the prominence of the blue reflection from the outer cell wall and therefore enhancing the visually blue appearance (Figure S3). 
Thus the colour of $V$. tinus fruits results from a combination of a physical nanostructure that selectively reflects blue wavelengths of light and a base layer of pigments that enhance the blue colour.

\section{A globular multilayer nanostucture composes the majority of the outer cell wall}

To characterize the nanostructures generating blue colour in $V$. tinus fruits, we used multiple electron microscopy techniques. Scanning electron microscopy of fresh tissue (Figure 2A) clearly shows the presence of a thick $(10-30+\mu \mathrm{m})$, multilayered structure, parallel to the surface of the fruit and embedded in the cell wall of the outermost epicarpal cells. A waxy cuticle ( 2-6 $\mu \mathrm{m})$ on top of the layered structure covers the fruit surface. The layered architecture occupies most of the outer cell wall in the region between the cuticle and the cellulose-rich primary cell wall. The layers range in thickness between 30-200 nm (Figure 2) and extend across the whole cell. Transmission electron microscopy (Figure 2) reveals that this architecture consists of many layers of small vesicles that differ in electron scattering power and refractive index from the matrix. Imaging by SEM and TEM (Figures 2, 3 \& S4) indicates that the matrix appears to contain key components of typical plant cell walls, namely cellulose and hemicelluloses. Staining with ruthenium red (Figures 3D \& S4A) shows a significant pectin content, and electron diffraction demonstrates the presence of cellulose by characteristic diffraction rings of the native cellulose crystal (Figure S4). Although the refractive index contrast layers are discrete and remain distinct from one another, considerable disorder is introduced by non-parallel neighbouring layers and the irregularity of their globular structure. Serial tomography (Figure 2E) of the epidermal cell wall reveals that these globular vesicles are organised in merged layers through which the cellulosic cell wall matrix remains connected by bridges and strings (Figure 2B; a 3D rotation model of this structure is available in the supplemental material Video S1).

\section{Evidence for the presence of lipids in the globular contrast phase}

We demonstrated that the globular multilayer structure in the $V$. tinus fruit epidermis is composed of lipids embedded in a cell wall matrix using a variety of methods. As solvency in nonpolar organic solvents is a well-defined hallmark of lipids, we imaged unfixed cryo ultrathin sections of the fruit epidermis before and after exposure to chloroform.[13] TEM images from the same sample region before (Figure $3 \mathrm{~A}$ ) and after (Figure 3B) chloroform exposure show that the globular structure has been removed by the treatment. In the latter image, the contrast of the globular multilayer phase is reduced and the empty structures within the matrix remain visible. In comparison, exposure to water did not alter the ultrastructure or image contrast of the globular multilayer, indicating that the material is extractable only with non-polar solvents. Secondly, when imidazole buffered osmium tetroxide, which binds to lipids, [14][15] was used during the chemical fixation process, the globular layers were preferentially stained (Figure 2 ) confirming their lipidic nature. In contrast, when ruthenium red was used (which binds to pectin[16]) the cell wall matrix was preferentially stained while the globular structure was washed away in the absence of imidazole buffer (Figure 3D). Additionally, a thin section of the epicarp was stained with Sudan Black B[17] which visibly stains lipids observed in the thickened cell wall in Figure S2D.

For all of the staining methods, we observed dark outlines around the globules (Figure 3E, Figure S4A). This could indicate the presence of a lipid membrane, which might be required the interface between hydrophobic molecules and the hydrophilic polysaccharides of the secondary cell wall.[18] 
Lipids comprise a diversity of molecular structures, generally classified as waxes, fats and oils, depending on their melting points.[19] Waxes are regularly found on the surface of the plant epidermis, making up the water-resistant waxy cuticle. Although waxes also include a diversity of molecular structures, [20] at least one prevalent component, alkanes,[21] are sufficiently indigestible to be a useful diet indicator in faecal samples.[22] In contrast, oils and fats are a vital nutritional resource as they contain much more energy per volume than is stored in starch or proteins. [15] Fats are generally found in large oil bodies inside cells of storage organs like seeds. In the case of $V$. tinus fruits, the close proximity of the globular structure to both the large energy-rich seed and the waxy external cuticle[23] makes the distinction between waxes and fats a particularly important one for understanding both the functional significance and developmental origins of the structure. In order to further identify whether the lipid globules are indigestible waxes or nutritious fats and oils, we used a variety of light-microscopy stains. Fixed sections were incubated with Nile Blue $A,[24]$ which stains the globule-rich region of the cell wall in $V$. tinus a blue or blue/purple-colour (Figure 3C). This indicates that the globules are more likely to be free fatty acids than cutin polymer, which would stain pink or red.[24]

Additionally, an electron diffraction diagram of the globular multilayer structure (Figure S4C-D) shows a sharp ring pattern, differing from the diagram of a cellulose cell wall with the characteristic two rings of cellulose crystals (Figure S4D). This pattern indicates that the lipid bodies are likely crystalline and therefore are homogenous monomeric lipids rather than polymerised molecules such as cutin, the waxy cuticle component, making them likely to be digestible. This raises the question of whether the lipids are derived from the cuticularisation process, arrested before polymerisation, [25] or derived from the internal nutritional lipids. Further extraction and characterisation would be required to ascertain the chemical composition and the developmental and evolutionary origins of these lipid bodies.

The biphasic organisation of the cell well maintains hydrophilic channels spanning the globular lipid structure. Given the high nutritionally relevant lipid content of the fruit,[3] and the very thin layer of flesh that composes the mesocarp, the proportion of lipid stored in the epicarp could be substantial, but this remains to be determined.

\section{Models of the optical reflectance from lipid globular multilayer}

In order to confirm that the observed mixed structure composed of a cellulosic matrix and layered lipid globules is responsible for the blue reflectance of $V$. tinus fruits, we modelled its optical response. To do this we investigated two mathematical models - (i) a 2D array of spheres, and (ii) averaging over many 1D biphasic multilayers. Disorder was introduced into the models and compared to experimental data.

An inverse design algorithm was used to model the structure in $2 \mathrm{D}$ as a series of globular accumulations; in Figure 4A-C each of the schematics corresponds to the adjacent modelled reflected spectrum.[26] This algorithm allows us to independently introduce different types of disorder into the globular multilayer by tailoring the size and structure factor, i.e. the Fourier transform of the positions of the particles. We studied the optical response of layered lipid globules with different amounts of variation in globule diameter (Figure 4A), disorder in the angle between adjacent globules (parameter $S p$, Figure 4B, no disorder produces a flat plane), and disorder in the average distance between adjacent globules (parameter $S k$, Figure $4 C$ ). The introduction of different types of disorder (Figure 4A-C) always produced the same effect on the optical response of the globular multilayer, namely reducing the peak intensity. 
Therefore, rather than dealing with each disorder element individually, the structure and material composition of the $V$. tinus cell wall was approximated as disordered 1D multilayers with refractive indices corresponding to cellulose $(n=1.55)[27]$ and a typical plant lipid $(n=1.47)$. [28] The model includes water immersion conditions, and the existence of a dark anthocyanin absorptive pigment underneath. The anthocyanin absorption spectrum used here is the cyanidin-3-glucoside extracted from bilberry (Vaccinium myrtillus), [29] which is one of the primary anthocyanins previously identified in $V$. tinus (the other being cyanidin 3-(200-xylosylglucoside)-5-glucoside).[12]

The statistical distribution of layer characteristics was measured from TEM cross-sections for initialisation in the multilayer model. The distributions of thicknesses of both materials are shown in Figure 4D. However, the effective model used a lower degree of disorder in layer thickness (s.d. $=25 \mathrm{~nm}$ rather than measured s.d. $=45 \mathrm{~nm}$ ) which is attributed to a small sample size, TEM artefacts[30] and long range order. The modelled reflectance using the averages over 1D multilayers is shown in Figure 4E.

Introducing disorder, as observed in cross-section measurements, into the model of a coherent ordered reflector broadens its reflectance band, in agreement with previous studies.[31] The broadband, asymmetric peak and angle-independence improves the fit of the resulting model to the real reflectance measured from $V$. tinus fruits, indicating that the model represents a good approximation to the $V$. tinus measurements. Due to its simplicity, the model is not meant to perfectly replicate the experimental measurements, but rather to indicate the validity of the disordered multilayer producing the optical response measured in $V$. tinus. The disorder in the multilayer in the form of boundaries of the globular multilayer that are non-perpendicular to the optical axis is consistent with the visibility of diffuse, wide-angle blue scattering observed in crosssection, as imaged in Figure S2E.

\section{Implications of lipid-based structural colour for the honest signalling of nutrition}

The colouration of $V$. tinus fruits is very striking to the human visual system. Comparison with blue tit spectral sensitivity (reproduced in Figure 1D[6]) shows that it falls well within the visually relevant range for relevant birds. The visual background for the fruit is predominantly the green leaves of $V$. tinus, the dominant pigment of which is chlorophyll. Chlorophyll has a broad spectral signature, with a peak at $550 \mathrm{~nm}$, and negligible reflectance below $500 \mathrm{~nm}$, making the colour of $V$. tinus fruits chromatically contrasted against foliage, as shown in Figure S3D. Birds are strongly responsive to visual stimuli,[32] and a predominant function of fruit colouration is to attract animals that carry out seed dispersal.[33][34] Thus, the lipid-based structural colour of $V$. tinus fruits could act as a visual signal for foraging birds.

Since birds likely use fruit colour to help them determine which fruits to consume, the colours of fruits may function as an honest signal of their nutritional reward. Honest signalling in fruits has been described, but the results are inconsistent. In some species in the Atlantic forest of Brazil, dark colouration corresponds with a carbohydrate-rich nutritional reward[35] whereas in other species in the Mediterranean, dark colouration has been associated with a lipid-rich nutritional reward. [36] The correlation between anthocyanin content and dark colour is thought to derive from shared biochemical pathways between anthocyanin and sugar synthesis.[35] Greater anthocyanin content typically makes fruits darker[37] although we know of no such biochemical explanation for the correlation between dark colouration and lipid content. 
We posit that blue fruit colour in $V$. tinus could serve as an honest signal of nutritional lipid content owing to fact that in this system lipids both create the colour and provide nutrition The lipids in the photonic structure are probably short-chain fatty acids, which are digestible by birds and thus contribute in part to the overall nutritional content. We did not determine what proportion of the high lipid nutritional reward in $V$. tinus fruits[3] is due to lipids in the photonic structure rather than lipids occurring elsewhere in the mesocarp.

Honest signalling systems are often thought to be expensive due to the cost of producing the signal. [36][38]. In this case, however, the interests of the signaller and the receiver are aligned: chromatic contrast enhances visibility for birds and signals a more valuable nutritional content to the bird, both features that are likely to increase dispersal. Although there is a cost associated with producing a blue photonic structure (the energy invested in making the lipids), that cost also provides direct benefits in the form of better advertising and better nutritional reward.

A disordered lipid multilayer as we report here in $V$. tinus has not been described previously in a biomaterial. However, the nanostructure in $V$. tinus may help us understand colouration in two other species with structurally coloured fruits (Elaeocarpus angustifolius[39] and Delarbrea michieana[40]), which show strong morphological parallels. For instance, $D$. michieana is described as having an iridosome, composed of "bubble-like" structures, that visually appears similar to the architecture we observe in $V$. tinus. [40] Should these other species prove to use a similar structure to that of $V$. tinus, it would provide an intriguing example of parallel evolution in distantly related plant lineages.

\section{Conclusions}

The architecture (a disordered multilayer reflector, generated by a biphasic structure of lipids and cellulose cell wall) in the epidermal cell walls of $V$. tinus fruits is unlike that of any previously described structurally coloured fruits, or any other known structurally coloured material. The coincidence of the striking colouration and high nutritional content of the coloured material itself suggests that it may be an honest signal of nutritional reward evolved as a result of biotic selection.

\section{Acknowledgements}

This work was supported by the EPSRC NanoDTC EP/G037221/1 (R.M.) and EPSRC EP/R513179/1 (R.M.), BBSRC David Phillips fellowship [BB/K014617/1] (S.V.), ERC SeSaME ERC-2014-STG H2020 639088 (S.V., Y.O., G.J.), a microMORPH Cross-Training Grant (M.S.A.), a Yale Institute for Biospheric Studies grant (M.S.A.), National Science Foundation (NSF)SF GRFP DGE-1122492 (M.S.A.), and NSF DBI 1907293 (M.S.A.). We would like to acknowledge the assistance of the Boulder Electron Microscopy Service in preparation and imaging the serial block-face, and the support of the Cambridge Advanced Imaging Centre and the NanoBio-ICMG platform (FR 2607) electron microscopy facility. We are grateful to Heather Whitney and Innes Cuthill for loan of equipment and to two anonymous referees for advice and comments which improved the manuscript.

\section{Author Contributions}

Conceptualisation, R. M., M.S.A., E.M., B.J.G., S.V.; Investigation: Optical Characterisation, R.M.; Electron Microscopy, M.S.A., Y.O., P.J.R., C.P., M.C. ; Numerical Modelling, R.M., G.J.; Composition Analysis, M.S.A., Y.O.; Formal Analysis, R.M., M.S.A.; Resources, R.M., M.S.A., E.M.; Writing - Original 
Draft, R.M.; Writing - Review \& Editing, All Authors; Visualisation, R.M., M.S.A., Y.O., G.J.; Supervision, B.J.G., M.D., S.V.;

\section{Declaration of Interests}

The authors declare no competing interests.

\section{Figure Legends}

Figure 1. Optical appearance of ripe $V$. tinus fruits. (A) A habitat photograph of ripe fruits on $V$. tinus shrub in natural light displaying a metallic blue appearance. An additional macro-photograph and UV-isolated image are shown in Figure S1. (B) Microscopic image of the fruit surface in reflected light, showing the angle-dependence of colour intensity over the curvature of each cell. Additional polarisation microscopy response and cross-sectional microscopy are shown in Figure S2. (C) Macroscopic UV-Vis reflectance spectrum taken by a double-ended probe and calibrated to a silver mirror reference. Additional macroscale spectra comparing fruit and leaf reflectance are included in Figure S3D. (D) Predicted blue tit (Parus caeruleus) spectral sensitivity from cone response, oil droplets and ocular media, reproduced from [6]. Sensitivities shown as a reference for the visual relevance of the fruit signal to potential fruit dispersers. The $\mathrm{x}$-axis is identical in both plots. The blue tit visual system is chosen for phylogenetic relatedness with a principal V. tinus frugivore, Sy/via atricapilla (Eurasian blackcap).

Figure 2. Electron microscopy and reconstruction of internal 3D architecture (A) Block-face SEM showing epidermal cell with internal cavity filled with pigmented contents. Most of the cell wall constitutes the globular multilayer. Labels: wc - waxy cuticle, gm - globular multilayer region, mc monophase (cellulosic) cell wall, cc-cell content. The dark red pigment is present in the cell content ' $c C^{\prime}$ ', and is seen in a light microscope cross-section in Figure S3E. A reduced magnification showing multiple cells is shown in Figure S4B. (B) TEM of the globular multilayer in the outer cell wall matrix; the cell outer surface is above the top edge. (C) Higher magnification TEM of globules shown in (B). (D) 3D reconstruction of globular layers reconstructed from TEM serial tomography using the isosurface function in iMod. The globular multilayer forms a merged plate-like structure rather than isolated globules. Full rotation video is shown in Video S1. (E) Reconstruction of globular inclusions using three joined serial tomograms parallel to the curved fruit surface. The plate-like structure parallel to the surface is visible, along with the bridges traversing it.

Figure 3. Identification of cell wall multilayer components. (A-B) A cryo-ultrathin section of the same portion of the globular multilayer region imaged before $(A)$ and after (B) extraction with chloroform, showing the removal of the electron-dense internal lipid phase in the globular layers. Arrows indicate the same location on the sample, which appears inverted due to the loss of stained lipid. (C) Nile Blue A staining of the fruit epicarp observed with transmitted light microscopy showing the cellulosic cell walls (dark blue), the outer cuticle (pink) and the mixed layer (purple) in the multilayer. (D) TEM image after ruthenium red staining indicates that pectin is present in the cell wall matrix, a higher magnification image is given in Figure S4(A). (E) TEM with lipid staining showing dark outlines around the globules. ew, epicuticular wax, wc - waxy cuticle, gm - globular multilayer region, $\mathrm{mc}$ - monophase (cellulosic) cell wall, $\mathrm{cc}-$ cell content.

Figure 4 Numerical models of multilayers and disorder. (A-C), Simulated optical response of $2 \mu \mathrm{m}$ thick, 2D models with different types of disorder as indicated in schematics (A1, B1, C1). (D) A histogram presenting data showing the distribution of layer widths for both cellulose and lipid layers, measured from analysis of TEM cross-section profiles. (E) Numerical model of the lipid-cellulosic 
structure as a 1D disordered multilayer: $58 \mathrm{~nm}$ (lipid layer), $67 \mathrm{~nm}$ (cellulose layer), s.d. $=25 \mathrm{~nm}$. The experimentally measured $\mathrm{V}$. tinus spectrum is shown again for comparison.

\section{STAR Methods}

Lead Contact: Silvia Vignolini sv319@cam.ac.uk

\section{Resource Availability}

This study did not generate new unique reagents, nor any unique datasets or code.

\section{Experimental Model and Subject Details}

$V$. tinus fruits were imaged with optical and electron microscopes ripening over the course of five years. We estimate fifteen separate occasions each for optical and electron microscopic analyses, the majority of which treated several samples from different fruits and individual plants. Samples were collected from private and college gardens around Cambridge and Bristol. TEM and SEM measurements were consistent across individuals and measurements. For the histogram in Figure 4D we used nine different TEM images from multiple sample fruits.

\section{Method Details}

\section{Optical Techniques}

For macro-spectroscopy (Figure 1C), deuterium - halogen tungsten lamp (DH-2000-BAL UV-VIS-NIR, Ocean Optics) illumination by a double ended fibre (QR400-7-UV-VIS, Ocean Optics) coupled to a Flame-S-UV-VIS miniature spectrometer was accessed by (Ocean Optics) software, and calibrated to a white standard (WS-1-SL, Ocean Optics) and a mirror reflectance tile (Avantes RS-2) for total intensity. The probe aperture was locked into a black aluminium mounting block obscuring stray light, $1 \mathrm{~cm}$ from the fruit surface.

In situ macro-spectroscopy of fruits and leaves (Figure S3D) was performed with an Ocean Optics USB2000 spectrometer illuminated by deuterium and halogen lamps, standardized with a Spectralon white reflectance standard (Ocean Optics, Dunedin, FL). Reflectance was measured $3 \mathrm{~mm}$ from the surface of the fruit or leaf.

For the optical microscopy (Figure 1B, S2-S3) and micro-spectroscopy (S2-S3), a Zeiss A1 microscope was used in reflection mode for optical measurements with a Zeiss Hal100 halogen lamp and a water-immersion objective lens (Zeiss W N-Achroplan 10x, 40x and 63x magnification). The image was captured on a CCD (IDS UI-3580LE-C-HQ) and accessed by uEye Cockpit user interface software. For polarisation measurements, incident (Zeiss 427710-9000) and reflection (Thorlabs $25 \mathrm{~mm}$ wire grid) polarisers were used for polarisation control. For micro-spectrometry, the reflected light was coupled via optical fibre (Avantes $50 \mu \mathrm{m}$ internal diameter) to a spectrometer (Avantes AvaSpecHS2048) and calibrated to a silver mirror (Thorlabs, PF10-03-P01).

The high magnification macroscale image in Figure S1A was taken using built-in LED full-ring illumination of a VHX-5000 Keyence digital microscope.

Photo in Figure $1 \mathrm{~A}$ was taken with a Sony DSLR- $\alpha 300$ and SAL30M28 macrolens. The UV-only photo Figure S1B was taken with a Nikon DSLR-D90 camera, 105mm f4.5 UV-Micro-APO lens (Coastal Optics) and Baader U-Filter 60nmHBW/320-380nm.

\section{Electron Microscopy}


Cryo-electron microscopy was carried out on fresh fruits sectioned, mounted on carbon glue and flash frozen by infiltration with liquid ethane at $-195^{\circ} \mathrm{C}$ and coated with $5 \mathrm{~nm}$ platinum using an FEI Varios 460 microscope with Quorum PP3010T cryo-SEM preparation and transfer system at Cambridge Advanced Imaging Centre.

For TEM, fresh tissue was prepared by immersion in Karnovsky's fixative for 1-14 days, dissected, and stained with osmium tetroxide for 2 hours. Tissue samples then experienced graduated infiltration with an ethanol-resin series to fixation in LR White resin and polymerised for 24 hours in vacuum at $60^{\circ} \mathrm{C}$.[41] A Leica UCT microtome and diamond knife was used to cut 70-100 nm sections. Sections were imaged on a Hitachi H-7650 transmission electron microscope (TEM) and a JEM 2100 Plus (Jeol) equipped with a RIO 16 CMOS camera (Thermo Fisher Scientific).

For serial tomography, serial semi-thick $(300 \mathrm{~nm}$ ) sections were cut using a Leica UCT umicrotome and diamond knife and mounted on a Formvar-coated slot grid. 15nm-diameter colloidal gold particles were attached to both sides of the grids to serve as fiducial markers in the tomographic reconstructions. Series of tilted images from $-60^{\circ}$ to $60^{\circ}$ angles were recorded every $1^{\circ}$ using a TECNAI F30 (FEI) operated at 300kV. Images from three sections were combined into a single, serial tomogram using the IMOD software package[42] and the lipid surfaces modelled using the IMOD isosurface function.

\section{Staining Histology}

Staining of the ultrathin sections of embedded samples was used for identification of structural composition in light microscopy (Nile Blue A, Sudan Black B) and electron microscopy (imidazole buffer, ruthenium red). Nile Blue $A$ stains neutral fats and waxes a red or pink colour but fatty acids blue [24] (Figure 3C). Sudan Black B stains lipids a dark blue or black colour (Figure S2D).[17],[43] Stains were applied for 60 minutes at room temperature (Nile Blue A, after washing in acetic acid for $10 \mathrm{~s}$ and isopropanol for $30 \mathrm{~s}$ ) or $60^{\circ} \mathrm{C}$ (Sudan Black B). Staining with Oil Red O was attempted but failed to penetrate the resin.

For chloroform extraction, transverse ultrathin sections of epicarp with a thickness of 80-90 nm were cut at cryogenic temperature $\left(-110^{\circ} \mathrm{C}\right)$ with a diamond knife on a Leica EM UC7 ultramicrotome and collected on carbon-coated copper grids. Grids with cryo-sections were exposed to chloroform under reflux for 1 hour. Both untreated and treated sections were then observed without further post staining using a TEM (JEM 2100Plus, JEOL) equipped with RIO16 CMOS camera (Gatan Inc.) and operated at $200 \mathrm{kV}$.

\section{Electron Diffraction}

Selected area electron diffraction (SAED) experiments were carried out with untreated cryo-sections and a TEM (JEM 2100 Plus) operated at $200 \mathrm{kV}$. A selected area aperture with a diameter of $500 \mathrm{~nm}$ was used. The SAED patterns were recorded on the RIO16 camera using the low-dose module of Serial EM software.

\section{Structural Analysis}

TEM profiles were analysed using Fiji[44] to extract the dimensional statistics of the two different refractive index layers. Nine globular multilayer cross-sectional TEM images were binarised and image intensity profiles measured, from which the width distributions of cellulose and lipid layers were taken.

\section{Numerical modelling}


2D structures with different types of disorder were generated using a recently developed inverse design algorithm discussed in detail in Jacucci et al.[26] Numerical simulations of the optical response of the generated structures were performed in Lumerical, a software using the finite difference time domain (FDTD) method. The simulations were performed using periodic boundary conditions in the direction perpendicular to the incoming beam - set as a plane wave - and perfect matching layer (PML) boundaries in the thickness direction. The numerical stability/convergence of the simulations was ensured by choosing an extensive simulated time of $0.1 \mathrm{~ns}$ and boundary conditions as stated. This ensured that the electric field in the structure decayed before the end of the calculation and that all the excitation light was either reflected or transmitted. Each of the presented curves was obtained averaging the optical simulations of five different ensembles of particles with identical parameters.

The disordered 1D multilayer was built with the jreftran[45] function in MATLAB. An average of 100 trials were taken of $1 \mathrm{D}$ multilayer stacks of 160 pitch repeats, with lipid and cellulose layers having widths 58 and $67 \mathrm{~nm}$ respectively, with a standard deviation of $25 \mathrm{~nm}$ and refractive indices $n=1.47$ and 1.55 respectively, taken from literature values as described in the main text. The upper refractive index boundary was matched to non-absorptive water $(n=1.33)$ and the lower to a complex refractive with light absorption taken from measurements of cyanidin-3-glucoside extracted from bilberry (Vaccinium myrtillus).[29] Angle of incidence was $0^{\circ}$.

\section{Quantification and Additional Analysis}

No further methods were used to determine whether the data met assumptions, as a statistical approach was not taken.

\section{Additional Resources}

Video S1: Movie showing 3D rotation of globular multilayer structure model. Related to Figure 2D. Video reconstructed from three sets of TEM serial tomography using the isosurface function in iMod. The single view shown in Figure 2D corresponds to a frame of this reconstruction.

This study did not generate any unique datasets or code.

\section{References}

1. Maryam I.S. Alkurdi, Jan Supuka, L.F., and Mária Bihunová (2014). Viburnum tinus L. as a new Mediterranean element for central Europe urban landscapes. IOSR J. Agric. Vet. Sci. 7, 50-58.

2. Thebaud, C., and Debussche, M. (1992). A Field Test of the Effects of Infructescence Size on Fruit Removal by Birds in Viburnum tinus. OIKOS 65, 391-394.

3. Debussche, M., Cortez, J., and Rimbault, I. (1987). Variation in Fleshy Fruit Composition in the Mediterranean Region : The Importance of Ripening Season, Life-Form, Fruit Type and Geographical Distribution. Oikos 49, 244-252.

4. Jordano, P. (1982). Migrant Birds Are the Main Seed Dispersers of Blackberries in Southern Spain. Oikos 38, 183-193.

5. Debussche, M., and Isenmann, P. (1992). A Mediterranean bird disperser assemblage: composition and phenology in relation to fruit availability. Rev. d'Ecologie (Terre la Vie) 47, 411-432.

6. Hart, N.S., and Vorobyev, M. (2005). Modelling oil droplet absorption spectra and spectral sensitivities of bird cone photoreceptors. J. Comp. Physiol. A Neuroethol. Sensory, Neural, 
7. Nevo, O., Valenta, K., Razafimandimby, D., Melin, A.D., Ayasse, M., and Chapman, C.A. (2018).

8. Dirr, M. (2007). Viburnums: Flowering shrubs for every season. (Portland, Oregon, USA: Timber Press).

9. Jordano, P., and Herrera, C.M. (1980). The frugivorous diet of blackcap populations Sy/via atracapilla wintering in Southern Spain. Short Commun. Br. Ornithol. Union, 502-507.

10. Herrera, C.M. (1981). Fruit food of robins wintering in southern spanish mediterranean scrubland. Bird Study 28, 115-122.

11. Devide, Z. (1986). Über die ursache des blauglanzes der früchte von Viburnum tinus L. [On the Causes of Blue Sheen of Viburnum tinus Fruit (non-translated)]. Acta Bot. Croat. 45, 97-100.

12. Jordheim, M., Giske, N.H., and Andersen, Ø.M. (2007). Anthocyanins in Caprifoliaceae. Biochem. Syst. Ecol. 35, 153-159.

13. Ohlrogge, J., and Browse, J. (1995). Lipid Biosynthesis. Plant Cell 7, 957-970.

14. Angermüller, S., and Fahimi, H.D. (1982). Imidazole-buffered osmium tetroxide: an excellent stain for visualization of lipids in transmission electron microscopy. Histochem. J. 14, 823835.

15. Huang, A.H.C. (1992). Oil bodies and oleosins in seeds. Annu. Rev. Plant Physiol. Plant Mol. Biol. 43, 177-200.

16. Anderson, C.T., Carroll, A., Akhmetova, L., Somerville, C., and S, M.B.C. (2010). Real-Time Imaging of Cellulose Reorientation during Cell Wall Expansion in Arabidopsis Roots. Plant Physiol. 152, 787-796.

17. Mcgee-russell, B.S.M., and Smale, N.B. (1963). On Colouring Epon-Embedded Tissue Sections with Sudan Black B or Nile Blue A for Light Microscopy. J. Cell Sci. s3-104, 109-115.

18. Murphy, D.J. (1993). Structure, function and biogenesis of storage lipid bodies and oleosins in plants. Prog. Lipid Res. 32, 247-280.

19. McNair, J. (1929). the Taxonomic and Climatic Distribution of Oils, Fats, and Waxes in Plants. Am. J. Bot. 16, 832-841.

20. Jetter, R., Schaffer, S., and Riederer, M. (2000). Leaf cuticular waxes are arranged in chemically and mechanically distinct layers: evidence from Prunus laurocerasus L. Plant, Cell Envinronment 23, 619-628.

21. Herbin, G.A., and Robins, P.A. (1969). Patterns of variation and development in leaf wax alkanes. Phytochemistry 8, 1985-1998.

22. Dove, H., and Mayes, R.W. (1996). Plant Wax Components: A New Approach to Estimating Intake and Diet Composition in Herbivores. J. Nutr. 126, 13-26.

23. Heredia, A. (2003). Biophysical and biochemical characteristics of cutin, a plant barrier biopolymer. Biochim. Biophys. Acta - Gen. Subj. 1620, 1-7.

24. Buda, G.J., Isaacson, T., Matas, A.J., Paolillo, D.J., and Rose, J.K.C.C. (2009). Three-dimensional imaging of plant cuticle architecture using confocal scanning laser microscopy. Plant J. 60, 378-385. 
25. Delude, C., Moussu, S., Joubès, J., Ingram, G., and Domergue, F. (2016). Ch. 12 Plant Surface Lipids and Epidermis Development. In Lipids in Plant and Algae Development, pp. 288-313.

26. Jacucci, G., Bertolotti, J., and Vignolini, S. (2019). Role of Anisotropy and Refractive Index in Scattering and Whiteness Optimization. Adv. Opt. Mater. 7.

27. Moon, R.J., Martini, A., Nairn, J., Simonsen, J., and Youngblood, J. (2011). Cellulose nanomaterials review: structure, properties and nanocomposites.

28. Kumar, S., Bhondekar, A.P., Jain, P., Bagchi, S., Sharma, A., Kumar, R., and Mishra, S. (2018). Artificial lipid membrane: Surface modification and effect in taste sensing. IOP Conf. Ser. Mater. Sci. Eng. 360.

29. David, I., Ştefănuţ, M.N., Căta, A., lenaşcu, I., Pop, R., Tănasie, C., and Balcu, I. (2009). Study of anthocyanins from Vaccinium Myrtillus L . frozen fruits. JAP\&T 15, 348-352.

30. Mollenhauer, H.H. (1993). Artifacts caused by dehydration and epoxy embedding in transmission electron microscopy. Microsc. Res. Tech. 26, 496-512.

31. Rengarajan, R., Mittleman, D., Rich, C., and Colvin, V. (2005). Effect of disorder on the optical properties of colloidal crystals. Phys. Rev. E - Stat. Nonlinear, Soft Matter Phys. 71, 1-11.

32. Valido, A., Schaefer, H.M., and Jordano, P. (2011). Colour, design and reward: Phenotypic integration of fleshy fruit displays. J. Evol. Biol. 24, 751-760.

33. Tiffney, B.H. (1984). Seed Size, Dispersal Syndromes, and the Rise of the Angiosperms: Evidence and Hypothesis. Missouri Bot. Gard. 71, 551-576.

34. Stournaras, K.E., Lo, E., Böhning-Gaese, K., Cazetta, E., Matthias Dehling, D., Schleuning, M., Stoddard, M.C., Donoghue, M.J., Prum, R.O., and Martin Schaefer, H. (2013). How colorful are fruits? Limited color diversity in fleshy fruits on local and global scales. New Phytol. 198, 617629.

35. Cazetta, E., Zumstein, L.S., Melo-Júnior, T.A., and Galetti, M. (2008). Frugivory on Margaritaria nobilis L.f. (Euphorbiaceae): poor investment and mimetism. Rev. Bras. Botânica 31, 303-308.

36. Schaefer, H.M., Valido, A., and Jordano, P. (2014). Birds see the true colours of fruits to live off the fat of the land. Proc. R. Soc. B Biol. Sci. 281.

37. Schaefer, H.M. (2011). Why fruits go to the dark side. Acta Oecologica 37, 604-610.

38. Számadó, S. (2011). The cost of honesty and the fallacy of the handicap principle. Anim. Behav. 81, 3-10.

39. Lee, D.W. (1991). Ultrastructural Basis and function of iridescent blue colour of fruits in Elaeocarpus. Nature 349, 260-262.

40. Lee, D.W., Taylor, G.T., and Irvine, A.K. (2000). Structural Fruit Coloration in Delarbrea michieana (Araliaceae). Int. J. Plant Sci. 161, 297-300.

41. Vignolini, S., Thomas, M.M., Kolle, M., Wenzel, T., Rowland, A., Rudall, P.J., Baumberg, J.J., Glover, B.J., and Steiner, U. (2012). Directional scattering from the glossy flower of Ranunculus: how the buttercup lights up your chin. J. R. Soc. Interface 9, 1295-1301.

42. Kremer, J.R., Mastronarde, D.N., and McIntosh, J.R. (1996). Computer visualization of threedimensional image data using IMOD. J. Struct. Biol. 116, 71-76.

43. Bayliss, O.B., and Adams, C.W.M. (1972). Bromine-Sudan Black : a general stain for lipids including free cholesterol. Histochem. J. 4, 505-515. 
44. Schindelin, J., Arganda-Carreras, I., Frise, E., Kaynig, V., Longair, M., Pietzsch, T., Preibisch, S., Rueden, C., Saalfeld, S., Schmid, B., et al. (2012). Fiji: An open-source platform for biologicalimage analysis. Nat. Methods 9, 676-682.

45. Divitt, S. (2020). jreftran - A layered thin film transmission and reflection coefficient calculator. MATLAB Cent. File Exch. Available at: https://www.mathworks.com/matlabcentral/fileexchange/50923-jreftran-a-layered-thinfilm-transmission-and-reflection-coefficient-calculator. 